\title{
Efeitos de um programa de intervenção terapêutico ocupacional em pacientes com hipertensão arterial sistêmica
}

\section{Effects of an occupational therapy intervention program on patients with systemic arterial hypertension}

\author{
Karoline Vitória Silva Rodrigues ${ }^{1}$, Yarima Silva Gomes de Castro ${ }^{2}$, \\ Enise Cássia Abdo Najjar ${ }^{3}$
}

http://dx.doi.org/10.11606/issn.2238-6149.v28i1p63-70

\begin{abstract}
Rodrigues KVS, Castro YSG, Najjar ECA. Efeitos de um programa de intervenção terapêutico ocupacional em pacientes com hipertensão arterial sistêmica. Rev Ter Ocup Univ São Paulo. 2017 jan.-abr.;28(1):63-70.
\end{abstract}

RESUMO: O estudo objetivou analisar os efeitos da intervenção de Terapia Ocupacional, no âmbito da educação em saúde, no conhecimento acerca da HAS e na adesão ao tratamento do paciente hipertenso. Trata-se de um estudo clínico, descritivo, com abordagem quanti-qualitativa. Participaram do estudo 7 pacientes hipertensos atendidos pelo programa HIPERDIA de uma Unidade Básica de Saúde de Belém, que apresentaram conhecimento precário e/ou baixa adesão ao tratamento da HAS. Os pacientes foram selecionados a partir da aplicação de dois questionários semiestruturados que objetivaram avaliar o conhecimento acerca da HAS, a adesão ao tratamento e a rotina ocupacional dos participantes. A pesquisa foi desenvolvida em três etapas: aplicação dos questionários, intervenção educativa em 13 sessões e re-aplicação dos questionários. A análise de dados foi desenvolvida de forma quanti-qualitativa. Os resultados apontaram uma ampliação em média de $76,4 \%$ dos níveis de conhecimento $\left(\mathrm{p}=0,0007^{*}\right)$ e de $77,81 \%$ nos níveis de adesão ao tratamento $(\mathrm{p}=0,0012 *)$ dos participantes após a realização da intervenção, com introdução de hábitos mais saudáveis na rotina ocupacional. Concluiu-se que o conhecimento dos participantes acerca de sua doença contribuiu para o aumento da adesão ao tratamento, evidenciando estreita relação entre rotina ocupacional e adesão ao tratamento. Constatou-se a eficácia da intervenção de Terapia Ocupacional na abordagem de educação em saúde junto ao paciente hipertenso.

DESCRITORES: Hipertensão; Educação em saúde; Terapia ocupacional.
Rodrigues KVS, Castro YSG, Najjar ECA. Effects of an occupational therapy intervention program on patients with systemic arterial hypertension. Rev Ter Ocup Univ São Paulo. 2017 Jan.-Apr.;28(1):63-70.

\begin{abstract}
The objective of this study was to analyze the effects of Occupational Therapy intervention on health education, knowledge about Systemic Arterial Hypertension (SAH) and adherence to treatment of hypertensive patients. This is a clinical, descriptive study, with quantitative-qualitative approach. Seven hypertensive patients attended by the HIPERDIA program of a Primary Health Unit in Belém, Brazil, participated in the study, who presented poor knowledge and/or low adherence to the treatment of hypertension. Patients were selected from the application of two semi-structured questionnaires that aimed to evaluate the knowledge about SAH, adherence to treatment and occupational routine of the participants. The research was developed in three stages: application of questionnaires, educational intervention in 13 sessions and re-application of questionnaires. Data analysis was developed in a quantitative-qualitative way. The results showed an average increase of $76.4 \%$ in the knowledge levels $\left(p=0.0007^{*}\right)$ and $77.81 \%$ in the levels of adherence to the treatment $\left(\mathrm{p}=0.0012^{*}\right)$ of participants after the intervention, with introduction of healthier habits in the occupational routine. It was concluded that the participants' knowledge about their disease contributed to the increase of adherence to the treatment, evidencing a close relationship between occupational routine and adherence to treatment. It was verified the effectiveness of the Occupational Therapy intervention in the approach of health education to the hypertensive patient.
\end{abstract}

KEYWORDS: Hypertension; Health education; Occupational therapy.

Este artigo é parte de Trabalho de Conclusão de Curso de Terapia Ocupacional da Universidade do Estado do Pará intitulado "Terapia Ocupacional e Hipertensão Arterial Sistêmica: uma abordagem em educação em saúde”. Apresentado como comunicação oral no Congresso Brasileiro de Terapia Ocupacional, Rio de Janeiro, 12-15 out. 2015. Fonte de auxílio: Pibic CNPq.

1. Terapeuta Ocupacional, Residente em Atenção à Saúde Cardiovascular da Universidade do Estado do Pará (UEPA), Belém, Pará, Brasil. Email: karoltorodrigues@hotmail.com

2. Terapeuta Ocupacional da Clínica Fisiocenter, Belém, Pará, Brasil. Email: yarimacastro21@hotmail.com

3. Docente do curso de Terapia Ocupacional - Universidade do Estado do Pará (UEPA), Belém, Pará, Brasil. Doutora em Teoria e Pesquisa do Comportamento pela Universidade Federal do Pará. Email: najjarenise@gmail.com

Endereço para correspondência: Departamento de Terapia Ocupacional. Travessa Perebebui, 2326 - Bairro do Marco, Belém, CEP: 66.625-460, Belém - PA. 


\section{INTRODUÇÃO}

Hipertensão Arterial Sistêmica (HAS)
constitui-se como o agravo mais comum e
de maior repercussão clínica na qualidade de vida do indivíduo ${ }^{1}$. Por ser uma patologia crônica, exige tratamento adequado e contínuo, de modo a contribuir para a prevenção de possíveis complicações e aumentar a qualidade de vida do paciente hipertenso ${ }^{2}$.

Para que o tratamento da HAS seja adequadamente realizado, evidencia-se a importância de uma equipe multiprofissional. A equipe deve atuar não somente no tratamento farmacológico, mas também no tratamento não medicamentoso, por meio de estratégias, como as utilizadas em educação em saúde, que podem aumentar a adesão ao tratamento da $\mathrm{HAS}^{3}$.

No que diz respeito à adesão ao tratamento, esta consiste no grau de correspondência entre o comportamento de uma pessoa e as recomendações acordadas com o profissional de saúde ${ }^{4}$.

A educação em saúde voltada para a HAS pode favorecer maior conhecimento da patologia e contribuir para mudanças comportamentais e de estilo de vida, por meio do desenvolvimento do autocuidado e da autonomia dos indivíduos frente a sua doença crônica. Além disso, promove a prevenção de enfermidades, como as doenças cardiovasculares, cerebrovasculares e renais, colaborando para melhor condição de vida .

Neste sentido, destaca-se o papel do terapeuta ocupacional na atenção básica, como profissional que favorece o entrelaçamento de ações de promoção de saúde, prevenção de agravos, tratamento e reabilitação. Neste campo de atuação, o terapeuta ocupacional considera os usuários como os protagonistas na produção de sua própria saúde .

No contexto das Doenças Crônicas Não Transmissíveis, o Terapeuta Ocupacional é um profissional especializados na prevenção, na modificação do estilo de vida e no envolvimento dos indivíduos no processo de gerenciamento de sua doença crônica, desenvolvendo estratégias de enfrentamento e mudanças de comportamentos e hábitos que se tornem parte de sua rotina ocupacional ${ }^{7}$.

Logo, considera-se a importância da atuação do terapeuta ocupacional junto ao paciente com HAS, como parte integral da equipe multiprofissional, em vista de este profissional ter sua atuação centrada no fazer humano e favorecer modificações de hábitos, rotinas e papéis ocupacionais, promovendo maior adesão ao tratamento ${ }^{8}$.

Este estudo teve como objetivo analisar os efeitos de um Programa de Atividades Terapêuticas Ocupacionais, no âmbito da educação em saúde, no conhecimento acerca da HAS e na adesão ao tratamento de pacientes com HAS.

\section{PROCEDIMENTOS METODOLÓGICOS}

Trata-se de um estudo clinico descritivo, com abordagem quanti-qualitativa. A pesquisa foi aprovada pelo Comitê de Ética em Pesquisa (CEP) da Universidade do Estado do Pará (UEPA), parecer de número 919.149, em dezembro de 2014.

Participaram sete pacientes com hipertensão arterial sistêmica, atendidos pelo programa HIPERDIA de uma Unidade Básica de Saúde de Belém. Todos os participantes assinaram o Termo de Consentimento Livre e Esclarecido.

Os critério de inclusão foram pacientes regularmente matriculados no programa HIPERDIA e que apresentaram scores inferiores a $60 \%$ na avaliação dos instrumentos aplicados, relacionados ao conhecimento da HAS e à adesão ao tratamento da doença. O programa HIPERDIA foi proposto pelo Ministério da Saúde em 2001, com os objetivos de cadastramento e acompanhamento de indivíduos acometidos por HAS e/ou diabetes mellitus9 9

Para a coleta de dados foram utilizados dois questionários semiestruturados, elaborado previamente para o estudo. O primeiro com onze questões objetivou avaliar duas dimensões: Conhecimento do participante a respeito da HAS (níveis pressóricos de um indivíduo hipertenso, sintomas da HAS, fatores de risco da HAS, complicações da HAS e cuidados no tratamento); e Adesão ao tratamento (cuidados com a alimentação, realização de atividade física, uso adequado da medicação, ingestão de bebida alcoólica, consumo de tabaco e vivência de situações de estresse). O segundo questionário com oito questões, buscou conhecer a rotina ocupacional dos participantes e verificar a percepção dos pacientes acerca da interferência das atividades cotidianas no tratamento da HAS. Contemplou quatro componentes: Atividades domésticas (arrumar a casa, varrer a casa, preparar as refeições, lavar a roupa, lavar a louça, dentre outros); Trabalho (exercício de atividade laboral remunerada, presença de estresse/prazer na atividade laboral); Lazer (realização de atividades de lazer, periodicidade semanal destas atividades); e Relações interpessoais (presença de conflitos familiares, frequência destes conflitos, existência de rede de apoio extrafamiliar).

Os questionários foram aplicados a 47 participantes. Destes, 24 preencheram os critérios de inclusão e foram convidados a participar do Programa de Atividades Terapêuticas Ocupacionais. Entretanto, apenas sete aceitaram o convite para participar do Programa. 
O Programa consistiu em 13 sessões, desenvolvidas no período aproximado de dois meses, com dois encontros semanais de aproximadamente 1 hora e meia de duração. Utilizaram-se estratégias de educação em saúde, com temáticas embasadas nas VI Diretrizes Brasileiras de Hipertensão - VI $\mathrm{DBH}^{3}$. O programa constou de: cinco encontros, em que se abordaram aspectos referentes ao conhecimento da patologia (características gerais da HAS, sintomas e fatores de risco, complicações, e tratamento); um encontro em que se analisou e refletiu com os participantes sobre a rotina ocupacional de cada paciente; seis encontros onde se abordou a adesão ao tratamento (controle do estresse psicoemocional, realização de atividade física e cuidados com a alimentação) e um encontro no qual se realizou o planejamento de nova rotina ocupacional juntamente com os participantes. Os encontros foram divididos em três momentos: no primeiro momento realizou-se o acolhimento dos participantes, apresentação da temática e do objetivo do encontro; no segundo momento ocorreu o desenvolvimento da atividade proposta; e no terceiro momento realizouse a troca de experiências sobre a atividade realizada. O Programa de Atividades objetivou, portanto, compartilhar informações relevantes para o tratamento da HAS, analisar e refletir acerca da rotina ocupacional dos participantes, sensibilizar os pacientes para a necessidade de incorporação de atividades de autocuidado para melhorar a adesão ao tratamento. As terapeutas ocupacionais durante todo o Programa de Atividades buscaram ser facilitadoras do processo de educação em saúde, abordando os participantes como protagonistas do seu próprio tratamento.

Como atividades, foram utilizadas atividades expressivas (recorte e colagem; montagem, teatro), atividades de relaxamento, atividades educativas (vídeos educativos, folders, construção de painel informativo, roda de conversa com nutricionista) e atividades de lazer (caminhada com participação e instrução de educador físico), com os objetivos de favorecer a descontração, a expressão, o conhecimento, a reflexão e a integração grupal.

Como recursos materiais, foram utilizados recursos de papelaria (papel A4, folhas de EVA, papel cartão, caneta esferográfica, caneta hidrocor, cola, tesoura, etc), figuras impressas ilustrativas, folders, som, caixa de som, data show, colchonetes, mesas e cadeiras. Com recursos humanos: as pesquisadoras do estudo, e também a participação de um educador físico e uma nutricionista em um encontro específico acerca da importância da atividade física e dos cuidados alimentares adequados para pacientes hipertensos, respectivamente.

Após o encerramento do grupo de intervenção os mesmos instrumentos foram reaplicados a fim de avaliar os efeitos do programa de intervenção no Conhecimento e na Adesão ao Tratamento.

A análise de dados foi desenvolvida de forma quantitativa e qualitativa, para melhor interpretação da realidade e maior alcance dos objetivos do estudo. $\mathrm{Na}$ análise estatística foram aplicados métodos descritivos e inferenciais. A análise dos dados dos questionários resultou em um escore para cada aspecto analisado, correspondendo a um ponto para cada alternativa correta. Para atribuição dos escores totais, as respostas foram convertidas em uma escala cuja pontuação variou de 0 (zero) a 23 (vinte e três) pontos para o aspecto Conhecimento e de 0 (zero) a 12 (doze) pontos para o aspecto Adesão.

Deste modo, no questionário relacionado ao Conhecimento, os participantes que alcançaram de 0 a 9 pontos corresponderam a $0 \%$ a $39,9 \%$ de respostas corretas e foram classificados em baixos níveis; os participantes que alcançaram de 0 a 14 pontos corresponderam a $40 \%$ a $59,9 \%$ e foram classificados em níveis médios; e os participantes que alcançaram de 15 a 23 pontos corresponderam a $60 \%$ a $100 \%$ de respostas corretas e foram classificados em altos níveis.

Quanto ao questionário relacionado à Adesão ao Tratamento, os participantes que alcançaram de 0 a 4 pontos corresponderam a $0 \%$ a $39,9 \%$ de cuidados adequados e foram classificados em baixos níveis; os participantes que alcançaram de 5 a 7 pontos corresponderam a $40 \%$ a $59,9 \%$ de cuidados adequados e foram classificados em níveis médios; e os participantes que alcançaram de 8 a 12 pontos corresponderam a $60 \%$ a $100 \%$ de cuidados adequados e foram classificados em altos níveis.

O teste t de Student para amostras pareadas foi utilizado para determinar a diferença entre as aplicações dos questionários antes e após a intervenção. Ficou previamente fixado o nível de significância alfa $=0.05$ para rejeição da hipótese de nulidade. O processamento estatístico foi realizado nos softwares GrafTable versão 2.0 e BioEstat versão 5.3. Os valores significantes foram assinalados por $(*)$.

Os dados qualitativos coletados por meio dos relatos livres dos participantes durante a intervenção realizada foram documentados pelas pesquisadoras em forma de registro em um diário de campo e submetidos à análise de conteúdo. Durante a análise realizada evidenciou-se o tema e as características associadas ao tema ${ }^{10}$, onde foram definidas três categorias analíticas: conhecimento da HAS, adesão ao tratamento e rotina ocupacional.

Para identificação dos participantes, optou-se em utilizar a letra $\mathrm{P}$ seguida de número, por exemplo, P1, $\mathrm{P} 2$ até $\mathrm{P} 7$. 


\section{RESULTADOS E DISCUSSÃO}

Dos 7 participantes, 6 eram do sexo feminino com idade entre 55 e 78 anos, destacando-se P1, P3, P4 e P5 com idade superior a 70 anos. Quatro participantes concluíram o ensino fundamental e quatro possuíam renda familiar entre 1 a 2 salários mínimos. A maioria, com exceção de P7, eram aposentados.

Em relação ao gênero, estudos demonstraram que mulheres tendem a procurar mais os serviços de saúde, o que implica no aumento das chances de detectarem a HAS precocemente, além disso, afirmam que a sobrevida é maior em mulheres quando comparadas a homens, estando assim mais propensas ao diagnóstico de doenças crônicas ${ }^{11}$.

Quanto à idade, alguns estudos apontam que os níveis tensionais tendem a aumentar com a idade, considerando valores maiores ou iguais a 14/9, sendo a prevalência de HAS superior a $60 \%$ na faixa etária acima de 60 anos de idade, como observado no presente estudo ${ }^{3,12}$.

$\mathrm{Na}$ análise dos fatores de risco presentes, P3 e P4 estavam com a pressão elevada, $15 / 8$ e 16/10 respectivamente. P4, P5 e P6 possuíam Diabetes Mellitus, P7 estava com obesidade grau 2 (IMC 35,06 ) e P3 com obesidade grau 3 (IMC 41,71). P1, P2 e P3 apresentaram colesterol e triglicerídeos acima dos níveis recomendados, conforme as VI $\mathrm{DBH}^{3}$. Observou-se, portanto que todos os indivíduos da pesquisa apresentaram pelo menos um fator de risco. No estudo realizado por Radocanovic et al. ${ }^{13} \mathrm{com}$ pacientes hipertensos os autores encontraram resultados similares, no qual todos os participantes com idade a partir de 50 anos apresentaram pelo menos um fator de risco cardiovascular e destes, $40 \%$ apresentaram cinco ou mais fatores de risco presentes.

Ao término do Programa de Atividades, detectouse que todos apresentaram a pressão arterial controlada, inclusive $\mathrm{P} 3$ e $\mathrm{P} 4$.

\section{- Conhecimento da HAS}

Quanto ao Conhecimento acerca da HAS, antes de iniciar o Programa de Atividades, os participantes P2, P3, P5, P6 e P4 apresentaram níveis baixos. P1 e P7 apresentaram níveis médios de Conhecimento. Os participantes desconheciam os valores pressóricos da HAS (P2, P3, P4, P5, P6, P7), os sintomas (P2, P5, P6), os fatores de risco (P2, P3, P6), as complicações (P3) e os cuidados com o tratamento (P2, P6).

Após a intervenção, o participante P5 foi o que mais se destacou apresentando um aumento de $83,9 \%$ no Conhecimento da HAS, seguidos de P2 e P6 que apresentaram aumento de $60,9 \%$. Os participantes P1, P3 e P4 apresentaram aumento acima de $30 \%$ no conhecimento. P7 que já possuía nível médio de Conhecimento da HAS, antes da intervenção, alcançou nível alto de conhecimento. Vale ressaltar que todos os participantes alcançaram nível alto de conhecimento ao final do Programa de Atividades com exceção de P3, como observado no Gráfico 1.

Gráfico 1 - Conhecimento dos participantes $(n=7)$ acerca da HAS antes e após o programa de atividades. Belém, PA, 2015

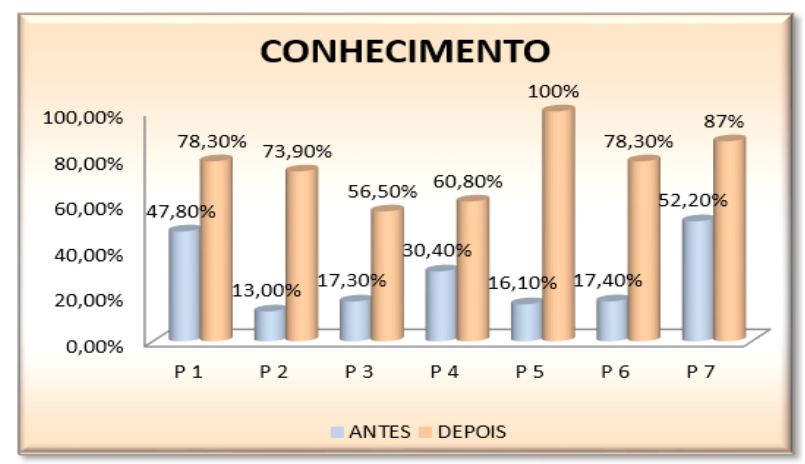

Destaca-se que os resultados em relação ao Conhecimento sobre HAS pelos sete pacientes, revelaram que houve aumento estatisticamente significante $\left(\mathrm{p}=0,0007^{*}\right)$ entre a avaliação antes $(27,7 \pm 16,2$ pontos $)$ e a avaliação depois ( $76,4 \pm 14,8$ pontos), portanto um aumento de 48,7 pontos.

Com o desenvolver das intervenções, notou-se que os participantes apresentavam cada vez mais interesse em conhecer a patologia e demonstraram compreender a importância deste conhecimento para o tratamento. Buscavam de modo independente, o acesso a novos conhecimentos acerca da HAS:

Eu nunca estudei a hipertensão. Mas ontem fui pesquisar na internet e vi que mais de quarenta milhões de brasileiros tem pressão alta. Fiquei surpreso (P7).

Antes do grupo eu não sabia nada da pressão alta. Hoje eu já estou sabendo. E isso é importante né?! Porque se eu não sei, como vou saber o que fazer ou se estou bem da minha pressão? (P2).

A importância do conhecimento dos pacientes com hipertensão sobre sua patologia, gravidade e cuidados relacionados ao tratamento é fator apontado na literatura como fundamental para a adesão, em vista de que o acesso às informações da HAS favorece a percepção de riscos à saúde e a importância do autocuidado ${ }^{14}$. 
Em pesquisa realizada na UBS do estado do Ceará ${ }^{15}$, os autores buscaram identificar o nível de conhecimento dos hipertensos acerca de sua patologia, de seus determinantes, complicações, bem como a adequação ao tratamento e hábitos de vida. Constataram que $90 \%$ dos indivíduos não souberam descrever o que é HAS, $65 \%$ não souberam citar os fatores de risco e $55 \%$ adequaram parcialmente seus hábitos de vida ao tratamento da hipertensão. Os achados deste estudo se assemelham aos resultados encontrados na presente pesquisa.

Ong et al. ${ }^{16}$ ressaltam que resultados favoráveis na avaliação do conhecimento dos pacientes com hipertensão decorrem provavelmente das informações adequadas fornecidas ao público sobre a doença, de investimento de treinamentos dos profissionais de saúde, da participação de equipes multidisciplinares no sistema de saúde local, de uma política mais agressiva no nível de cuidados primários e da participação do paciente em seu processo de cuidado. Observou-se nesta pesquisa, que as informações adequadas apresentadas durante o Programa de Atividades e a participação do paciente foram fatores que contribuíram para os resultados favoráveis deste estudo.

\section{- Adesão ao Tratamento da HAS}

Em relação à adesão ao tratamento relatado pelos participantes antes do Programa de Atividades, identificouse que quanto ao "uso correto da medicação", apenas P4 relatou não fazer uso adequadamente. No que se refere aos "cuidados com a alimentação", P2, P3, P4 e P5 relataram não realizar adequadamente. Quanto à "realização de atividade física", P1, P3, P4, P6 e P7 relataram não praticar atividade física. Em relação à "ausência de situações de estresse", P2, P4, P6 e P7 relataram vivenciar estas situações em seu cotidiano. O paciente $\mathrm{P} 4$ destacou que vivenciava situações de estresse em uma frequência de mais de três vezes durante a semana.

Após a realização do Programa de Atividades, a partir dos relatos dos participantes, identificou-se que todos apresentaram aumento na adesão ao tratamento. P2, P3 P5 relataram mudanças nos "cuidados com a alimentação", referindo diminuir a frequência do consumo de alimentos com sal, fritura e gordura. Em relação à "realização de atividade física", P1 e P7, que antes não realizavam, relataram desempenhar. Quanto à "vivência de situações de estresse", P4, P6 e P7 relataram evitar o enfrentamento de situações estressantes como, por exemplo, as discussões com familiares e a sobrecarga do trabalho. Sobre o uso adequado da medicação, P4, que antes não desenvolvia, passou a desenvolver corretamente. Os resultados podem ser visualizados no Gráfico 2.
Gráfico 2 - Adesão ao tratamento dos participantes $(\mathrm{n}=7)$ antes e após ao programa de atividades. Belém, PA, 2015

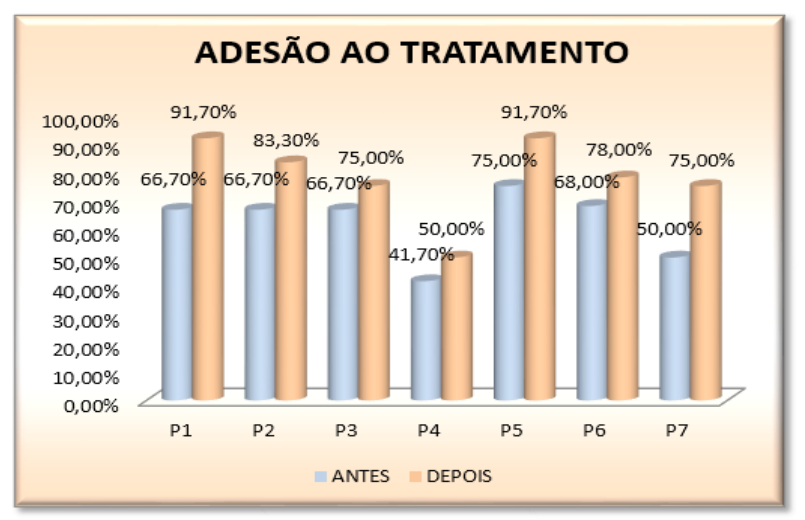

Os resultados apontaram que, em relação à adesão ao tratamento da HAS relatada pelos sete pacientes, houve aumento estatisticamente significante $\left(\mathrm{p}=0,0012^{*}\right)$ entre a avaliação antes ( $62,7 \pm 77,8$ pontos) e a avaliação depois $(76,4 \pm 14,8$ pontos), portanto um aumento de 15,1 pontos.

Durante a execução do Programa de Atividades, observou-se que de forma gradual, os participantes buscaram introduzir em seu cotidiano hábitos mais saudáveis e relatavam ter mudanças nos cuidados com a saúde antes não desenvolvidos:

Já estou percebendo a minha pressão mais comportada. Acho que porque desde o começo do grupo estou tentando ter mais cuidado com a minha alimentação. (P6)

É dificil, mas aos pouquinhos estou evitando mais as discussões com o meu filho... tem que evitar né?! Se não um dia ainda vou infartar com isso! (P4)

Graças a Deus agora eu comecei a caminhar todos os dias e ainda estou levando a minha esposa que é diabética. Vinte minutinhos por dia é rapidinho... (P7)

Modificações nos hábitos alimentares se tratam de costumes adquiridos e presentes desde a infầncia, relacionado à origem étnica e à condição socioeconômica do indivíduo, não se caracterizando, portanto, como hábitos de fácil mudança $\mathrm{a}^{17}$. Baldissera ${ }^{18}$ revela em seus estudos com pacientes hipertensos que os cuidados relacionados à alimentação adequada foi o aspecto mais negligenciado, com apenas $2,78 \%$ de adesão. O presente estudo reforça os achados da autora, pois a maior parte dos participantes, P2, P3, P4, P5 e P7 relataram dificuldades em desenvolver modificações ou restrições na alimentação desde o diagnóstico da HAS. 
Em relação à realização de atividade física, Serafim et al. ${ }^{19}$ esta não é uma prática comumente vivenciada por grande parte dos pacientes hipertensos, apesar de sua estreita relação com o controle da pressão arterial ${ }^{19}$. Neste contexto, Souza et al. ${ }^{20}$ revelam em seus estudos que mais da metade dos participantes não realizavam atividade física. Este estudo detectou resultados similares, pois cinco participantes, P1, P3, P4, P6 e P7 não desenvolviam tal cuidado.

Sobre o estresse psicoemocional, Reza e Nogueira ${ }^{21}$ detectaram em estudo realizado, que $82 \%$ dos participantes hipertensos se consideravam pessoas nervosas e estressadas, e que as situações que mais lhe causavam estresse, eram as relacionadas com as situações familiares ou do lar, as atividades do trabalho ou aquelas ligadas aos problemas sociais. Resultados similares foram encontrados neste estudo, no qual quatro participantes, P2, P4, P6 e P7, relataram a dificuldade de não conseguir evitar a vivência de situações de estresse no dia-a-dia.

Unger e Parati ${ }^{22}$ apontam que a vivência do estresse psicoemocional contribui para a hipertensão arterial sustentada, como se identificou nos relatos da paciente $\mathrm{P} 4$, que apresentou a PA elevada antes da participação no programa.

Verifica-se, deste modo, que realizar mudanças nos hábitos e no estilo de vida são dificuldades vivenciadas pelos pacientes com doenças crônicas. Entretanto, identificou-se que todos os participantes, passaram a desenvolver algum cuidado relacionado ao tratamento antes não desenvolvido, quer seja relacionado à alimentação, e/ ou à pratica de atividade física, e/ou ao controle do estresse e/ou ao uso adequado da medicação. Destaca-se que o participante P7 foi o que mais apresentou modificações na adesão, conseguindo desempenhar cuidados relacionados à realização de atividade física em frequência diária e ao controle do estresse psicoemocional, anteriormente não desenvolvidos.

\section{- Rotina Ocupacional}

Em relação à rotina ocupacional, os seis participantes aposentados - P1, P2, P3, P4, P5 e P6 consideravam o trabalho doméstico que realizavam como atividade laboral. Detectou-se também, que P3, P4, P5, P6 e P7 não realizavam atividades de lazer.

Na percepção dos participantes $\mathrm{P} 2, \mathrm{P} 3, \mathrm{P} 4, \mathrm{P} 5$ e P6 a rotina ocupacional contribuía para a adesão ao tratamento da HAS, enquanto P1 referiu que não interferia na adesão, enquanto $\mathrm{P} 7$ relatou que dificultava a adesão, devido à sobrecarga da atividade laboral e a frequência de vivências de situações de estresse.

Entretanto, a análise detalhada dos dados relacionados à rotina ocupacional possibilitou observar que o participante $\mathrm{P} 4$ realizava trabalho doméstico estressante e vivenciava conflitos familiares frequentes duas a três vezes por semana, que poderiam dificultar a manutenção do tratamento. Portanto não possuía uma rotina ocupacional que contribuísse para a adesão adequada ao tratamento, como havia classificado no questionário. Além disto, $\mathrm{P} 4 \mathrm{e}$ P7 apresentavam dificuldades significativas em desenvolver, de forma adequada, os cuidados relacionados à alimentação, em decorrência da rotina familiar e do cotidiano do trabalho.

Por outro lado, P5 demonstrou durante a realização do Programa de Atividades, que apresentava uma rotina ocupacional organizada, que contribuía para o desenvolvimento de um tratamento adequado, relatando discernimento em relação à importância dos cuidados com a saúde, buscando praticá-los cotidianamente.

Durante o desenvolvimento do Programa de Atividades, os demais pacientes P1, P2, P3 e P6 relatavam apresentar dificuldades em realizar mudanças na rotina ocupacional, as quais já estavam habituados há muito tempo.

Após a intervenção, observou-se que os participantes $\mathrm{P} 6$ e $\mathrm{P} 7$ passaram a desenvolver atividades de lazer pelo menos uma vez por semana. P7 relatou também, que passou a controlar melhor o estresse psicossocial no ambiente de trabalho. $\mathrm{O}$ trabalho que era considerado antes do Programa de Atividades como estressante, ao final da intervenção foi classificado como pouco estressante.

P7, após a intervenção, passou a organizar sua rotina ocupacional de modo que pudesse desempenhar diariamente atividade física:

Agora estou me organizando melhor. Acordo um pouco mais cedo, saio para caminhar todos os dias, e ainda estou levando minha esposa que está aderindo a tudo junto comigo... e é bom que ela é diabética né!?. Ai volto, fico na taberna sozinho enquanto ela faz o nosso almoço... está tudo melhor assim, para nós dois. (P7)

Diante das mudanças realizadas na rotina ocupacional, o participante $\mathrm{P} 7$ alcançou um aumento de $25 \%$ no aspecto adesão ao tratamento.

Quanto à participante $\mathrm{P} 4$, esta relatou que os conflitos familiares diminuíram de intensidade, passando a classificar sua frequência em uma vez por semana. Apontou que tal fato contribuiu para a melhora do seu humor durante o dia, e inclusive, para concentração no desenvolvimento de algumas atividades cotidianas importantes, como a lembrança em tomar o remédio de forma adequada:

... a gente está brigando menos graças a Deus. Não é aquela
coisa, mas está melhor. Estou procurando não me estressar
muito, evitar algumas coisas, ignorar outras... É melhor 
assim, fico menos aperreada no dia. Me concentro para fazer as minhas coisas e não fico pensando na raiva o tempo todo. Lembro até de tomar meu remédio certinho. (P4)

Quanto aos pacientes P1, P2, P3 e P5, estes não apresentaram mudanças em sua rotina ocupacional após o Programa de Atividades. Entretanto, demonstraram maior comprometimento e empenho nos cuidados com o tratamento, tais como maiores cuidados com a alimentação, realização de atividade física e controle do estresse.

No que diz respeito ao hábito de praticar atividades de lazer, percebeu-se que a maior parte dos pacientes não só desconheciam a real importância desta atividade para a saúde e a qualidade de vida, como também não a realizava. Após a intervenção, dos sete participantes, apenas dois passaram a realizar atividade de lazer.

Em pesquisa realizada por Baldissera e Bueno ${ }^{23}$ sobre lazer e HAS, as autoras encontraram achados que diferem deste estudo. Os pacientes entrevistados destacaram que o lazer é uma forma de enfrentar a solidão, pois favorece a socialização, o entusiasmo pela vida e a saúde mental. Além disso, o lazer foi considerado como estratégia de enfrentamento e tratamento da hipertensão.

Quanto à rotina ocupacional, estudos realizados por Nair et al. ${ }^{24}$ revelam que tais atividades possuem influência significativa na adesão ao tratamento do indivíduo hipertenso, indicando que a organização da rotina ocupacional contribui positivamente para a adesão aos cuidados necessários ao tratamento.

Os dados deste estudo corroboram o estudo de Nair et al. ${ }^{24}$ apontando também para uma estreita relação entre rotina ocupacional e adesão ao tratamento da HAS. Evidencia também, que as ocupações da rotina ocupacional podem exercer impacto no controle dos níveis pressóricos.

\section{CONCLUSÃO}

A pesquisa apontou para uma ampliação estatisticamente significante dos níveis de conhecimento e de adesão ao tratamento dos participantes após a realização do Programa de Atividades. O estudo confirmou que o conhecimento dos participantes com HAS acerca de sua doença contribui para o aumento da adesão ao tratamento, bem como evidenciou uma estreita relação entre rotina ocupacional e adesão ao tratamento.

O estudo revela, no âmbito da Terapia Ocupacional, a importância do enfoque na rotina ocupacional do paciente hipertenso, pois a partir da análise cuidadosa com adequação das atividades desta rotina foi possível alcançar maiores índices de adesão ao tratamento. Dessa forma, ressalta-se que o estudo favoreceu a manutenção da saúde e o controle da pressão arterial, contribuindo para a prevenção de complicações a curto prazo dos participantes.

Além disto, o estudo permitiu constatar, a importância das orientações das VI DBH quanto ao valor da intervenção de educação em saúde no acompanhamento de pacientes hipertensos.

No contexto da Atenção Primária à Saúde, a pesquisa evidencia, portanto, a eficácia da intervenção de Terapia Ocupacional na abordagem de educação em saúde com pacientes com HAS.

Aponta-se para a necessidade de investigar de modo mais aprofundado, em estudos futuros, a relação entre conflitos familiares, Hipertensão Arterial Sistêmica e rotina ocupacional, em decorrência da prevalência dos relatos dos participantes a respeito da vivência desses conflitos e das consequentes dificuldades na adesão ao tratamento.

\section{REFERÊNCIAS}

1. Boing AC, Boing AF. Hipertensão Arterial Sistêmica: o que nos dizem os sistemas brasileiros de cadastramentos e informação em saúde. Rev Bras Hipertensão. 2007;14(2):84-8. Disponível em: http://departamentos. cardiol.br/dha/revista/14-2/06-hipertensao.pdf.

2. Alves BA, Calixto AATF. Aspectos determinantes da adesão ao tratamento de hipertensão e diabetes em uma Unidade Básica de Saúde no interior paulista. J Health Sci Inst. 2012;30(3):255-60. Disponível em:http://www.unip. br/comunicacao/publicacoes/ics/edicoes/2012/03 jul-set/ V30_n3_2012_p255a260.pdf.

3. Sociedade Brasileira de Cardiologia. Sociedade Brasileira de Hipertensão. Sociedade Brasileira de Nefrologia. VI Diretrizes Brasileiras de Hipertensão. Arq Bras Cardiol. 2010;95(1 supl.1):1-51. Disponível em: http://publicacoes. cardiol.br/consenso/2010/Diretriz_hipertensao_ERRATA.pdf.

4. Organización Mundial de La Salud. Adherencia a lostratamientos a largo plazo: pruebas para la acción. Washington, D.C.; 2004. Available from: http://www. paho.org/hq/index.php?option=com_docman\&task $=$ doc view\&gid $=18722 \&$ Itemid $=270$. 
5. Ribeiro AG, Cotta RMM, Silva LS, Ribeiro SMR, Dias CMGC, Mitre SM, et al. Hipertensão arterial e orientação domiciliar: o papel estratégico da saúde da família. Rev Nutr. 2012;25(2):271-82. http://dr.doi.org/10.1590/S141552732012000200009 .

6. ROCHA EF, SOUZA CCBX. Terapia ocupacional em reabilitação na atenção primária à saúde: possibilidades e desafios. Rev Ter Ocup Univ São Paulo.. 2011;22(1):36-44. http://dx.doi.org/10.11606/issn.2238-6149.v22i1p36-44.

7. American Occupational Therapy Association. The role of occupational therapy in primary care. Am J Occup Ther. 2014;68:25-33. doi:10.5014/ajot.2014.686S06.

8. Duarte GP, Uchôa-Figueiredo LR. A vida cotidiana e a qualidade de vida de pacientes atendidos na atenção primária de saúde. Cad Ter Ocup UFSCar. 2010;18(1):19-33. Disponível em: http://www.cadernosdeterapiaocupacional. ufscar.br/index.php/cadernos/article/view/330/265.

9. Brasil, Ministério da Saúde. DATASUS: Hiperdia. Brasília; 2001. Disponível em: http://datasus.saude.gov.br/sistemase-aplicativos/epidemiologicos/hiperdia.

10. Bardin L. Análise de conteúdo. São Paulo: Edições 70; 2011.

11. Mendes GS, Moraes CF, Gomes L. Prevalência de hipertensão arterial sistêmica em idosos no Brasil entre 2006 e 2010. Rev Bras Med Fam Comunidade. 2014;9(32):2738. Disponível em: http://www.rbmfc.org.br/rbmfc/article/ view/795/641.

12. Silva LR, Oliveira EAR, Lima LHO, Formiga LMF, Sousa ASJ, Silva RN. Fatores de risco para hipertensão arterial em policiais militares do centro-sul piauiense. Rev Baiana Saúde Publica. 2014;38(3):679-92. http://dr.doi.org/10.5327/ Z0100-0233-2014380300014.

13. Radovanovic CAT, Santos LA, Carvalho MDB, Marcon SS. Hipertensão arterial e outros fatores de risco associados à doenças cardiovasculares em adultos. Rev Lat-Am Enfermagem. 2014;22(4):22-4. http://dr.doi.org/10.1590/ S0103-2100201300020012.

14. Nolêto SMG, Silva SM, Barbosa CO. Conhecimento dos hipertensos sobre a doença. RBCEH. 2011;8(3):324-32. http://dr.doi.org/10.1590/0104-1169.3447.2442.

15. Lima ER, Barros ARC, Oliveira CAN. Percepção dos clientes hipertensos acerca das complicações da hipertensão arterial sistêmica. Rev Interfaces Saúde Humanas Tecnol. 2014;2(5):1-9. http://dr.doi.org/10.16891/2317.434X.96.

16. Ong KL, Cheung BM, Man YB, Lau CP, Lam KS. Prevalence, awareness, treatment, and control of hypertension among United States adults 1999-2004. Hypertension. 2007;49:6975. doi: 10.1161/01.HYP.0000252676.46043.18.

17. Oliveira TL, Miranda LP, Fernandes OS, Caldeira AP. Eficácia da educação em saúde no tratamento não medicamentoso da hipertensão arterial. Acta Paul Enferm. 2013;26(2):179-84. http://dr.doi.org/10.1590/ S0103-21002013000200012.

18. Baldissera VDA. Análise da adesão ao tratamento nãofarmacológico anti-hipertensivo entre usuários de um centro de saúde escola [dissertação]. Maringá: Universidade Estadual de Maringá; 2005.

19. Serafim TS, Jesus ES, Pierin A. M. Influence of knowledge on health life style in the control of hypertensive. Acta Paul Enferm. 2010;23:658-64. http://dx.doi.org/10.1590/S010321002010000500012 .

20. Souza CS, Stein AT, Bastos Gan, PellandaLC .Controle da pressão arterial em hipertensos do Programa Hiperdia: estudo de case territorial. Arq Bras Cardiol. 2014;102(6):571-8. http://dr.doi.org/10.5935/abc.20140081.

21. Reza CG, Nogueira MS. O estilo de vida de pacientes hipertensos de um programa de exercício aeróbico: estudo na cidade de Toluca, México. Esc Anna Nery Rev Enferm. 2008;12(2):265-70. http://dx.doi.org/10.1590/ S1414-81452008000200010.

22. Unger T, Parati G. Acute stress and long-lasting blood pressure elevation: a possible cause of established hypertension? J Hypertens. 2005;200:261-3. http://journals. lww.com/jhypertension/Citation/2005/02000/Acute_stress and_long_lasting_blood_pressure.4.aspx.

23. Baldissera VDA, Bueno SMV. O lazer e a saúde mental das pessoas hipertensas: convergência na educação para a saúde. Rev Esc Enferm USP. 2012;46(2):380-7. http://dr.doi. org/10.1590/S0080-62342012000200016.

24. Nair KV, Belletti JJ, Allen RR, McQueen RB, Sassen JJ, Vande Griend J, et al. Understanding barriers to medication adherence in the hypertensive population by evaluating responses to a telephone survey. Patient Prefer Adherence. 2011;5:195-206. doi: 10.2147/PPA.S18481.

Recebido em: 18.05 .16

Aceito em: 19.01.17 